\title{
MODULAR UNITS AND THE $q$-DIFFERENCE EQUATIONS OF SELBERG
}

\author{
AMANDA FOLSOM
}

\begin{abstract}
In this paper we present a subgroup of modular units that arise naturally from analytic solutions to higher order $q$-recurrence equations given by Selberg in his work generalizing the Rogers-Ramanujan identities. Further, we express these modular units in terms of Siegel functions as considered by Kubert and Lang, and show they generate the group of all units of the modular curves $X(\ell)$ with cuspidal support on $\pi^{-1}(\infty)$, where $\pi: X(\ell) \rightarrow X_{0}(\ell)$ is the canonical projection.
\end{abstract}

\section{Introduction.}

For any $\ell \in \mathbb{N}$, let $\mathcal{F}_{\ell}$ be the modular function field with respect to the principal congruence subgroup $\Gamma(\ell)=\left\{\gamma \in \mathrm{SL}_{2}(\mathbb{Z}) \mid \gamma \equiv 1 \bmod \ell\right\}$ defined over $\mathbb{Q}\left(\zeta_{\ell}\right)$, where $\zeta_{\ell}=e^{2 \pi i / \ell}$. The invertible elements in the integral closure of the ring $\mathbb{Q}[j] \subset \mathcal{F}_{\ell}$ form a group $U_{\ell}$, the modular unit group of level $\ell$, where

$$
j=j(\tau)=q^{-1}+744+196884 q+\cdots
$$

is the classical modular invariant. In this paper we present a subgroup of modular units for primes $\ell \geq 5$

$$
U_{\ell}^{C}:=\left\langle\left\{r_{\ell, j}(\tau) \mid 1 \leq j \leq(\ell-3) / 2\right\}\right\rangle \subset U_{\ell}
$$

with generators $r_{\ell, j}(\tau), 1 \leq j \leq(\ell-3) / 2$ defined in $\S 2$ using functional solutions to higher order $q$-recurrence equations found in the work of Selberg [12]. These units may be viewed as higher level analogues to a widely studied function, the RogersRamanujan continued fraction, defined by

$$
r(\tau)=\frac{q^{1 / 5}}{1+} \frac{q}{1+} \frac{q^{2}}{1+} \frac{q^{3}}{1+\ldots},
$$

convergent for $\tau$ in the upper half complex plane $\mathcal{H}$. We develop this analogy in 1.2 and $\S 2$, but first discuss an algebraic characterization of the groups $U_{\ell}^{C}$.

1.1. Kubert-Lang. The work of D. Kubert and S. Lang during the latter half of the 1970's, culminating in the book Modular Units [7], developed the algebraic theory, as well as the function theory, of the modular units. For a historical account of the Kubert-Lang theory, one may wish to see "Lang's Work on Modular Units and Frobenius Distributions" by D. Rohrlich in [6]. A major result of the Kubert-Lang theory is the complete characterization of the modular units in terms of Siegel functions, which we discuss in more detail in $\S 3$. In $\S 4$ we provide an expression for the modular units $r_{\ell, j}$ in terms of Siegel functions as considered by Kubert and Lang. We then use this

Received by the editors September 1, 2008. Revision received June 22, 2009. 
expression to give a characterization of the unit group $U_{\ell}^{C}$ as the group of all units of the modular curve $X(\ell)$ with cuspidal support on $\pi^{-1}(\infty)$, where $\pi: X(\ell) \rightarrow X_{0}(\ell)$ is the canonical projection. Once we have established such a factorization in terms of Siegel functions, our method of proof of this characterization of the groups $U_{\ell}^{C}$ follows in the spirit of methods of proof developed by Kubert and Lang in [7].

1.2. Rogers-Ramanujan. In addition to the algebraic characterization of the modular unit groups $U_{\ell}^{C}$, we also develop an analogy between the $r_{\ell, j}$ and the RogersRamanujan function $r(\tau)$, discovered first by Rogers in 1894 [9], and later independently by Ramanujan in 1913, and Schur in 1917 [11]. Although we present the Rogers-Ramanujan function here as an example of a modular unit, $r(\tau)$ has more notably been an object of study due to a wealth of associated analytic properties. These include various related $q$-series identities, consequences within partition theory, and far reaching combinatorial interpretations (see [2], for example). Many of the fundamental properties associated to $r(\tau)$ rely upon the fact that one may regard $r(\tau)$ as arising from the $q$-recurrence

$$
R(z)=R(z q)+z q R\left(z q^{2}\right) .
$$

That is, we may write

$$
r(\tau)=q^{1 / 5} R(q) / R(1) .
$$

The $q$-recurrence (4) has a known analytic solution [10] given by

$$
R(z)=\sum_{n \geq 0} z^{n} q^{n^{2}}(q ; q)_{n}^{-1}
$$

where $(a ; q)_{k}=\prod_{j=0}^{k-1}\left(1-a q^{j}\right)$ for integers $k \geq 1$, and $(a ; q)_{0}=1$. A celebrated result associated to $r(\tau)$ is the pair of Rogers-Ramanujan identities,

$$
\begin{aligned}
& R(1)^{-1}=\prod_{n \geq 1}\left(1-q^{5 n-1}\right)\left(1-q^{5 n-4}\right) \\
& R(q)^{-1}=\prod_{n \geq 1}\left(1-q^{5 n-2}\right)\left(1-q^{5 n-3}\right),
\end{aligned}
$$

which give an infinite product representation for the series (6) evaluated at $z=1$ and $z=q$. Various proofs have emerged in the literature for the identities (7) and (8), originally due to Rogers and (independently) Ramanujan, whose proofs rely upon the use of theta functions [10]. The identities may also be interpreted combinatorially (see for example [1]). By (5), (6), (7) and (8), one obtains a $q$-product expansion for $r(\tau)$. With this, one may show that $r(\tau)$ is in fact a modular function on $\Gamma(5)$, and also a modular unit. In what follows, we consider the fields $\mathcal{F}_{\ell}, \ell \geq 5$, and provide more general proofs of the above facts about $r(\tau)$ as a special case of the main theorem in $\S 4$. We also point the interested reader to the 2005 work of W. Duke [3] on the Rogers-Ramanujan continued fraction $r(\tau)$, who proves some beautiful properties of the function $r(\tau)$, its special values, and related functions. In [5], we also examine many other properties of the modular units $r_{\ell, j}$ of this paper. 


\section{The $q$-difference equations of Selberg.}

To define higher order modular unit groups analogous to $U_{5}^{C}=\langle r(\tau)\rangle$ generated by the Rogers-Ramanujan continued fraction $r(\tau)$, we seek other appropriate modular functions on the curves $X(\ell)=(\Gamma(\ell) \backslash \mathcal{H})^{*}$. Due to the fact that $r(\tau)$ has a continued fraction expansion and may be viewed as originating from a certain $q$-recurrence, one might hope that if analogous functions exist, that they exhibit similar traits. We indeed form such families for the curves $X(\ell)$ of higher genus $g>0$ in what follows, yet will see that these functions do not possess a continued fraction expansion, but rather an expression that may be viewed as a generalization of a continued fraction expansion. To describe this, we begin with the observation of Sylvester that a continued fraction may be expressed as the limiting value of ratios of $n^{\text {th }}$ order determinants. That is,

$$
a_{0}+\frac{b_{1}}{a_{1}+\frac{b_{2}}{a_{2}+\frac{b_{3}}{a_{3}+}}}=\left|\begin{array}{ccccc|ccccc}
a_{0} & b_{1} & 0 & 0 & \cdots \\
-1 & a_{1} & b_{2} & 0 & \cdots \\
0 & -1 & a_{2} & b_{3} & \cdots \\
0 & 0 & -1 & a_{3} & \cdots \\
\vdots & \vdots & \vdots & \vdots & & \vdots \\
\vdots & \vdots & & & & 0 & 0 & 0 & \cdots \\
0 & a_{1} & b_{2} & 0 & \cdots \\
0 & -1 & a_{2} & b_{3} & \cdots \\
0 & 0 & -1 & a_{3} & \cdots \\
\vdots & \vdots & \vdots & \vdots &
\end{array}\right|^{-1}
$$

where the successive ratios of order $n$ determinants are the partial convergents of the continued fraction. One finds that the Rogers-Ramanujan continued fraction may be expressed as

$$
r(\tau)=q^{1 / 5}\left|\begin{array}{ccccc||ccccc|}
1 & 0 & 0 & 0 & \cdots & 1 & q & 0 & 0 & \cdots \\
0 & 1 & q^{2} & 0 & \cdots & -1 & 1 & q^{2} & 0 & \cdots \\
0 & -1 & 1 & q^{3} & \cdots & 0 & -1 & 1 & q^{3} & \cdots \\
0 & 0 & -1 & 1 & \cdots & 0 & -1 & 1 & \cdots \\
\vdots & \vdots & \vdots & \vdots & & \vdots & \vdots & \vdots & \vdots &
\end{array}\right|
$$

To generalize this let $A^{(n)}$ be the order $n$ determinant with entries $a_{i, j}, 1 \leq i, j \leq n$, with $B^{(n)}$ defined similarly. Provided the limit exists, definethe ratio of two infinite determinants by

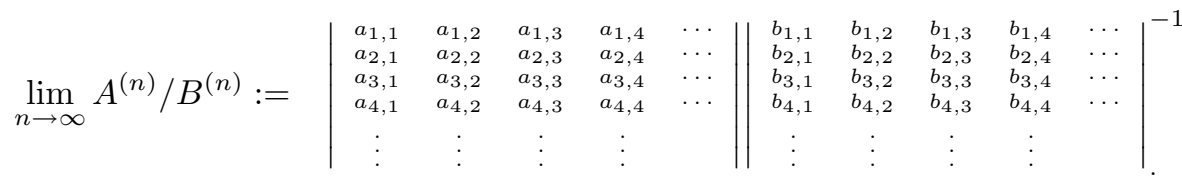

With hopes of constructing analogous modular functions to (9) of higher level, we form the infinite determinant $S_{k}(z)$ of width $k+1$

$$
S_{k}(z):=\left|\begin{array}{ccccccccc}
s_{k, 0}(z) & s_{k, 1}(z) & s_{k, 2}(z) & \ldots & s_{k, k-1}(z) & 0 & 0 & 0 & \cdots \\
-1 & s_{k, 0}(z q) & s_{k, 1}(z q) & s_{k, 2}(z q) & \cdots & s_{k, k-1}(z q) & 0 & 0 & \cdots \\
0 & -1 & s_{k, 0}\left(z q^{2}\right) & s_{k, 1}\left(z q^{2}\right) & s_{k, 2}\left(z q^{2}\right) & \cdots & s_{k, k-1}\left(z q^{2}\right) & 0 & \cdots \\
\vdots & \vdots & \vdots & \vdots & \vdots & \vdots & \vdots & \vdots
\end{array}\right| .
$$


One may deduce from (11) the higher order $q$-recurrences satisfied by the functions $S_{k}(z)$

$$
\sum_{m=0}^{k} s_{k, m-1}(z) S_{k}\left(z q^{m}\right)=0
$$

where we let $s_{k,-1}(z)=-1$. This can be done by first expanding $S_{k}(z)$ along the top row, then simplifying the determinant minors that appear, making use of the fact that columns eventually are of the form $(-1,0,0, \ldots)^{t}$. In doing so, one will see that these minors do indeed equal $S_{k}\left(z q^{m}\right), 0 \leq m \leq k$, and that (12) holds. We must define the functions $s_{k, j}(z)$ appropriately so that (12) yields an appropriate analytic solution $S_{k}(z)$. To do so, let

$$
\begin{aligned}
s_{k, 2 n}(z):= & (-1)^{n} z^{n k} q^{\delta(k, n)}(q ; q)_{n}^{-2} \\
& \times \sum_{j=0}^{\left.\frac{k+1}{2}\right\rfloor-n-1} z^{j} q^{j(n+1)}\left(q^{j+1} ; q\right)_{n}\left(q^{\left\lfloor\frac{k+1}{2}\right\rfloor-(j+n)} ; q\right)_{n} \\
s_{k, 2 n-1}(z):= & (-1)^{n+1} z^{n k-\left\lfloor\frac{k}{2}\right\rfloor} q^{\delta^{\prime}(k, n)}(q ; q)_{n-1}^{-2}\left(1-q^{n}\right)^{-1} \\
& \times \sum_{j=0}^{\left\lfloor\frac{k}{2}\right\rfloor-n} z^{j} q^{j n}\left(q^{j+1} ; q\right)_{n}\left(q^{\left\lfloor\frac{k}{2}\right\rfloor-(j+n)+1} ; q\right)_{n-1}
\end{aligned}
$$

for $n \geq 0$ in (13), and $n \geq 1$ in (14), where

$$
\begin{aligned}
\delta(k, n) & =(2 k+1) \frac{n^{2}+n}{2}-n\left\lfloor\frac{k+1}{2}\right\rfloor \\
\delta^{\prime}(k, n) & =(2 k+1) \frac{n^{2}-n}{2}+n\left\lfloor\frac{k+1}{2}\right\rfloor,
\end{aligned}
$$

and

$$
\begin{aligned}
& \lfloor\alpha\rfloor=\max _{n \in \mathbb{Z}}\{n \leq \alpha\} \\
& \lceil\alpha\rceil=\min _{n \in \mathbb{Z}}\{n \geq \alpha\} .
\end{aligned}
$$

As given in (13) and (14), the functions $s_{k, m}(z)$ are due to A. Selberg [12]. In the case $k=2$, the $q$-recurrence (12) reduces to (4), with $S_{2}(z)=R(z)$. With this choice of $s_{k, j}$, an analytic solution to the $q$-recurrence (12) exists, and is given in [12] by

$$
S_{k}(z)=\sum_{n=0}^{\infty}(-1)^{n} z^{k n} q^{(2 k+1) \frac{n^{2}+n}{2}-k n}\left(1-z^{k} q^{(2 n+1) k}\right) \frac{(z q ; q)_{n}}{(q ; q)_{n}} \prod_{m \geq 1}\left(1-z q^{m}\right)^{-1} .
$$

Selberg uses these functions to give certain identities that are analogous to the Rogers-Ramanujan identities (7) and (8), and also to give various $q$-continued fraction identities. In contrast to their use in [12], we use the functions $s_{k, j}(z)$ and $S_{k}(z)$ to define groups of modular units $U_{\ell}^{C}$. 
2.1. Defining the $r_{\ell, j}$. To define the modular units $r_{\ell, j}$, we first recall the Galois action on the modular function fields $\mathcal{F}_{\ell}$. The natural action of the group $\Gamma(1):=$ $\mathrm{SL}_{2}(\mathbb{Z}) \subset \mathrm{M}_{2}(\mathbb{Z})$ on the fields $\mathcal{F}_{\ell}$ is given by

$$
\gamma \cdot f(\tau)=f\left(\gamma^{-1} \tau\right)
$$

where $f=f(\tau) \in \mathcal{F}_{\ell}$. The group $\mathrm{GL}_{2}(\mathbb{Z} / \ell \mathbb{Z})$ also acts on $\mathcal{F}_{\ell}$ as follows. Given $d \in(\mathbb{Z} / \ell \mathbb{Z})^{*}$, let $\gamma_{d}=\left(\begin{array}{ll}1 & 0 \\ 0 & d\end{array}\right)$ act on $\mathbb{Q}\left(\zeta_{\ell}\right)$ by

$$
\gamma_{d} \cdot \zeta_{\ell}=\zeta_{\ell}^{d}
$$

If $f \in \mathcal{F}_{\ell}$ has $q$-series expansion given by $f(\tau)=\sum_{n=m}^{\infty} a_{n} q^{n / \ell}$, the action of $\gamma_{d}$ extends to $\mathcal{F}_{\ell}$ by

$$
\gamma_{d} \cdot f(\tau)=\sum_{n=m}^{\infty}\left(\gamma_{d} \cdot a_{n}\right) q^{n / \ell}
$$

The matrices $\gamma_{d}, d \in(\mathbb{Z} / \ell \mathbb{Z})^{*}$, together with $\mathrm{SL}_{2}(\mathbb{Z} / \ell \mathbb{Z})$, generate $\mathrm{GL}_{2}(\mathbb{Z} / \ell \mathbb{Z})$, and the group actions given by (16) and (17) define a Galois action on the modular function fields via the isomorphism

$$
\operatorname{Gal}\left(\mathcal{F}_{\ell} / \mathcal{F}_{1}\right) \stackrel{\pi}{\cong} \mathrm{GL}_{2}(\mathbb{Z} / \ell \mathbb{Z}) /\{ \pm 1\}
$$

We point out for later use that $\mathrm{GL}_{2}(\mathbb{Z} / \ell \mathbb{Z})$ has the following decomposition

$$
\mathrm{GL}_{2}(\mathbb{Z} / \ell \mathbb{Z}) \cong C_{2}(\ell) G_{\infty}(\ell),
$$

where $C_{2}(\ell)$ is the degree two Cartan group (whose elements may be represented by primitive pairs $\bmod \ell$ ), and $G_{\infty}(\ell)$ is the isotropy subgroup of the vector $(1,0)^{t}$. Using (15), we first define the functions $r_{\ell, 1}$ and $r_{\ell, 2}$.

Definition. For prime $\ell=2 k+1 \geq 5$, let

$$
r_{\ell, 1}(\tau)=(-1)^{k-1} q^{\frac{-k(k-1)}{2 \ell}} \frac{S_{k}(1)}{S_{k}(q)}
$$

and for prime $\ell=2 k+1 \geq 7$, let

$$
r_{\ell, 2}(\tau)=(-1)^{k-2} q^{\frac{-(k+1)(k-2)}{2 \ell}} \frac{S_{k}(1)-q^{k-1} S_{k}\left(q^{2}\right)}{S_{k}(q)} .
$$

For $m \in(\mathbb{Z} / \ell \mathbb{Z})^{*}$, we let

$$
\sigma_{m}:=\left(\begin{array}{cc}
m & 0 \\
0 & 1
\end{array}\right) \in \pi\left(\operatorname{Gal}\left(\mathcal{F}_{\ell} / \mathcal{F}_{1}\right)\right)
$$

and for $\ell=2 k+1$, let

$$
\Psi_{\ell}=\Psi_{\ell}(\tau):=-q^{\frac{(3 \ell-4)(\ell-3)}{24 \ell}} \frac{\eta(\tau)}{\eta(\ell \tau)} S_{k}(q) .
$$


Next we define for any positive integer $j$ satisfying $1 \leq j \leq(\ell-3) / 2$, the functions

$$
r_{\ell, j}(\tau):=\sigma_{k+1-j}\left(i \Psi_{\ell}\right) / i \Psi_{\ell}
$$

where the Dedekind $\eta$-function $\eta(\tau)=\Delta^{1 / 24}(\tau)$ is the $24^{\text {th }}$ root of the Discriminant function, and is given by

$$
\eta(\tau)=q^{1 / 24} \prod_{n \geq 1}\left(1-q^{n}\right) .
$$

We will see in $\S 4$ that the definition given in (24) coincides with (20) and (21) for $j=1,2$.

Remark. We remark that there is a way to appropriately define modular units $r_{\ell, j}$ for all $j, 1 \leq j \leq(\ell-3) / 2$ when $\ell$ is odd and not necessarily prime. (See also [5].)

\section{Modular units and Siegel functions.}

3.1. The Kubert-Lang theory. A major result of Kubert and Lang in their development of the theory of the modular units is the explicit characterization of the modular units of prime power level $\ell$ in terms of Siegel functions. The Siegel functions are constructed using the Klein forms, $\mathfrak{t}_{L}(z)$, which are defined by a lattice $L \subseteq \mathbb{C}$, and are functions of $z \in \mathbb{C}$. Equivalently, we may write $\mathfrak{t}_{L}(z)=\mathfrak{t}_{a}(\tau)$ if $L=\mathbb{Z} \tau+\mathbb{Z}$, and $z=a_{1} \tau+a_{2} \in \mathbb{C}, a_{1}, a_{2} \in \mathbb{R}$. With the latter notation, the Klein forms are defined by

$$
\mathfrak{t}_{a}(\tau)=e^{-\eta_{a}(\tau) a \cdot(\tau, 1) / 2} \sigma_{a}(\tau),
$$

where $\sigma_{a}$ and $\eta_{a}$ are the classical Weierstrass functions. Using known properties of the Weierstrass $\sigma_{a}$ and $\eta_{a}$ functions, one can verify that the Klein forms satisfy the following properties

$$
\begin{aligned}
\mathfrak{t}_{\lambda L}(\lambda z) & =\lambda \mathfrak{t}_{L}(z), \\
(c \tau+d) \mathfrak{t}_{a}(\gamma \tau) & =\mathfrak{t}_{a \gamma}(\tau), \\
\mathfrak{t}_{a+b}(\tau) & =\epsilon(a, b) \mathfrak{t}_{a}(\tau) .
\end{aligned}
$$

In (27)-(29), $\lambda \in \mathbb{C}^{*}, \gamma \in \Gamma(1), b=\left(b_{1}, b_{2}\right) \in \mathbb{Z}^{2}$, and the constant $\epsilon(a, b)$ is given by

$$
\epsilon(a, b)=(-1)^{b_{1} b_{2}+b_{1}+b_{2}} e\left(\left(b_{2} a_{1}-b_{1} a_{2}\right) / 2\right),
$$

where $e(\omega):=e^{2 \pi i \omega}$. We note in particular that $(27)$ implies

$$
\mathfrak{t}_{-a}(\tau)=-\mathfrak{t}_{a}(\tau)
$$

The Klein forms are used to define the Siegel functions, given by

$$
g_{a}(\tau)=\mathfrak{t}_{a}(\tau) \eta(\tau)^{2},
$$

where $\eta(\tau)$ is the Dedekind $\eta$-function. We point out the following transformation law.

Lemma 1. Let $a=\left(a_{1}, a_{2}\right) \in \mathbb{Q}^{2} \backslash \mathbb{Z}^{2}$. Then

$$
g_{\left(a_{1}, a_{2}\right)}(-1 / \tau)=-i g_{\left(a_{2},-a_{1}\right)}(\tau) \text {. }
$$


Proof. Using the definition (30) of the Siegel function $g_{a}$, the lemma follows from the transformation properties

$$
\begin{aligned}
\eta^{2}(-1 / \tau) & =-i \tau \eta^{2}(\tau) \\
\tau \mathfrak{t}_{\left(a_{1}, a_{2}\right)}(-1 / \tau) & =\mathfrak{t}_{\left(a_{2},-a_{1}\right)}(\tau) .
\end{aligned}
$$

We state the fundamental result of Kubert-Lang characterizing the modular units in terms of Siegel functions in the following Theorem.

Theorem K. (Kubert, Lang) Let $\ell=p^{n}$, where $p$ is prime, $p \neq 2,3, n \in \mathbb{N}$. Then the modular units of level $\ell$ (modulo constants) consist of products

$$
\prod_{a} g_{a}^{m(a)}
$$

where $a=\left(\frac{a_{1}}{\ell}, \frac{a_{2}}{\ell}\right) \in \frac{1}{\ell} \mathbb{Z}^{2}, a \notin \mathbb{Z}^{2}$, and the exponents $m(a) \in \mathbb{Z}$ satisfy the quadratic relations

$$
\sum_{a} m(a) a_{1}^{2} \equiv \sum_{a} m(a) a_{2}^{2} \equiv \sum_{a} m(a) a_{1} a_{2} \equiv 0 \bmod \ell
$$

and the condition $\sum_{a} m(a) \equiv 0 \bmod 12$.

Using the product expansion for the classical $\Delta$ and $\sigma$ functions, one has the following product formula for the Siegel functions:

$$
g_{a}(\tau)=-q^{\frac{1}{2} \mathbf{B}_{2}\left(\frac{a_{1}}{\ell}\right)} e\left(a_{2}\left(a_{1}-1\right) / 2\right)\left(1-q^{\frac{a_{1}}{\ell}} e\left(\frac{a_{2}}{\ell}\right)\right) \prod_{n=1}^{\infty}\left(1-q^{n+\frac{a_{1}}{\ell}} e\left(\frac{a_{2}}{\ell}\right)\right)\left(1-q^{n-\frac{a_{1}}{\ell}} e\left(-\frac{a_{2}}{\ell}\right)\right)
$$

where $\mathbf{B}_{2}(z)$ is the second Bernoulli polynomial defined by

$$
\mathbf{B}_{2}(z)=z^{2}-z+\frac{1}{6}
$$

One may verify by the Galois action described previously, the product formula (34), and the action (28), that the Galois group $\operatorname{Gal}\left(\mathcal{F}_{\ell} / \mathcal{F}_{1}\right)$ acts on the Siegel functions by multiplication on the indices. That is, for $\sigma \in \operatorname{Gal}\left(\mathcal{F}_{\ell} / \mathcal{F}_{1}\right)$,

$$
g_{a}^{\sigma}=g_{a \cdot \sigma} .
$$

In statement $i i$ of the main theorem in $\S 4$ we give an expression in terms of Siegel functions of the forms $r_{\ell, j}$.

3.2. Defining the unit groups $U_{\ell}^{+}$. The final characterization with respect to the forms $r_{\ell, j}$ we present in this paper (given in Theorem 1 iii) is the determination of the modular unit groups they generate. To do so, we define the subset $\mathcal{A}_{\ell}$ by

$$
\mathcal{A}_{\ell}:=\left\{\beta \mid \beta \in \pi_{\ell}^{-1}(\infty)\right\},
$$

where $\pi_{\ell}$ is the canonical projection

$$
\pi_{\ell}: X(\ell) \longrightarrow X_{0}(\ell)
$$


where

$$
\begin{aligned}
X_{0}(\ell) & \cong\left(\Gamma_{0}(\ell) \backslash \mathcal{H}\right)^{*} \\
\Gamma_{0}(\ell) & =\left\{\gamma=\left(\begin{array}{ll}
a & b \\
c & d
\end{array}\right) \in \Gamma(1) \mid \gamma \equiv\left(\begin{array}{ll}
* & * \\
0 & *
\end{array}\right) \bmod \ell\right\} .
\end{aligned}
$$

We use $\mathcal{A}_{\ell}$ to define the following subgroup of the modular unit group

$$
U_{\ell}^{+}:=\left\{f \in U_{\ell} \mid \operatorname{supp}(\operatorname{div}(f)) \subseteq \mathcal{A}_{\ell}\right\}
$$

where $\operatorname{supp}(x)$ denotes the support of $x$, and $\operatorname{div}(x)$ denotes the divisor of $x$.

\section{The main theorem.}

\section{Theorem 1.}

$i$. For primes $\ell=2 k+1 \geq 5$, the functions $r_{\ell, j}(\tau), 1 \leq j \leq(\ell-3) / 2$, are modular units of level $\ell$.

ii. The modular units $r_{\ell, j}$ have the following expressions in terms of Siegel functions:

$$
\begin{aligned}
r_{\ell, j}(\tau) & =(-1)^{(k-j)} e\left(-\frac{k(k-j)}{2 \ell}\right) \prod_{s=0}^{\ell-1} g_{((k+1-j) / \ell, s / \ell)}(\tau) / g_{(1 / \ell, s / \ell)}(\tau) \\
& =(-1)^{k-j} \prod_{s=-k}^{k} g_{((k+1-j) / \ell, s / \ell)}(\tau) / g_{(1 / \ell, s / \ell)}(\tau) .
\end{aligned}
$$

iii. For primes $\ell \geq 5$, the subgroup $U_{\ell}^{+}$defined in (41) of the modular unit group generated by those units with divisors supported at the cusps $\beta \in \pi_{\ell}^{-1}(\infty)$ is equal to the group $U_{\ell}^{C}$ defined in (2) generated by the modular units $r_{\ell, j}, 1 \leq$ $j \leq(\ell-3) / 2$. That is,

$$
U_{\ell}^{+}=U_{\ell}^{C}
$$

4.1. Proof of Theorem $1 i$ and $i i$. To show the functions $r_{\ell, j}$ as defined in (20), (21) and (24) are modular units of level $\ell$, we first establish the following Proposition.

Proposition 1. Let $k \geq 2$ be an integer, and let $\ell=2 k+1$. Then we have

$$
\begin{aligned}
S_{k}(1) & =\prod_{n \geq 1} \frac{\left(1-q^{\ell n-k}\right)\left(1-q^{\ell n-(k+1)}\right)\left(1-q^{\ell n}\right)}{\left(1-q^{n}\right)} \\
S_{k}(q) & =\prod_{n \geq 1} \frac{\left(1-q^{\ell n-1}\right)\left(1-q^{\ell n-(\ell-1)}\right)\left(1-q^{\ell n}\right)}{\left(1-q^{n}\right)} \\
S_{k}(1)-q^{k-1} S_{k}\left(q^{2}\right) & =\prod_{n \geq 1} \frac{\left(1-q^{\ell n-(k-1)}\right)\left(1-q^{\ell n-(k+2)}\right)\left(1-q^{\ell n}\right)}{\left(1-q^{n}\right)} .
\end{aligned}
$$


One of the tools required to prove Proposition 1 is the following product identity $\theta\left[\begin{array}{c}\epsilon \\ \epsilon^{\prime}\end{array}\right](\tau):=\sum_{n \in \mathbb{Z}} e\left(\frac{1}{2}\left(n+\frac{\epsilon}{2}\right)^{2} \tau+\left(n+\frac{\epsilon}{2}\right) \frac{\epsilon^{\prime}}{2}\right)=$

$$
e\left(\frac{\epsilon \epsilon^{\prime}}{4}\right) q^{\epsilon^{2} / 8} \prod_{n \geq 1}\left(1-q^{n}\right)\left(1+e\left(\frac{\epsilon^{\prime}}{2}\right) q^{n-\frac{1+\epsilon}{2}}\right)\left(1+e\left(\frac{-\epsilon^{\prime}}{2}\right) q^{n-\frac{1-\epsilon}{2}}\right),
$$

where $\epsilon, \epsilon^{\prime} \in \mathbb{R}$. The identity (48) may derived from Jacobi's triple product formula

$$
\sum_{n=-\infty}^{\infty} q^{n^{2}} z^{2 n}=\prod_{n=1}^{\infty}\left(1-q^{2 n}\right)\left(1+q^{2 n-1} z^{2}\right)\left(1+q^{2 n-1} z^{-2}\right) .
$$

(A proof of (48) appears in the book of Farkas and Kra, [4].)

Proof of Proposition 1. We first prove (45), which is straightforward. The proofs of (46) and (47) below are similar, although are more involved. The proof of (46) involves collapsing a sum of 4 sums on $\mathbb{Z}^{\geq 0}$ into only one sum on $\mathbb{Z}$, and the proof of (47) involves collapsing a sum of 10 sums on $\mathbb{Z}^{\geq 0}$ into only one sum on $\mathbb{Z}$.

Proof of (45). Beginning with the definition of $S_{k}(z)$ in (15), we find

$$
\begin{aligned}
\prod_{n \geq 1}\left(1-q^{n}\right) S_{k}(1)=\sum_{n \geq 0} & (-1)^{n} q^{(2 k+1)\left(n^{2}+n\right) / 2-k n} \\
& +\sum_{n \geq 0}(-1)^{n+1} q^{(2 k+1)\left(n^{2}+n\right) / 2-k n+(2 n+1) k} .
\end{aligned}
$$

We denote the two sums on the right hand side of (50) by $\Sigma_{1}$ and $\Sigma_{2}$ (i.e. $\prod_{n \geq 1}(1-$ $\left.\left.q^{n}\right) S_{k}(1)=\Sigma_{1}+\Sigma_{2}\right)$. We replace $n$ by $-m-1$ in $\Sigma_{2}$ and find

$$
\Sigma_{2}=\sum_{m \leq-1}(-1)^{m} q^{(2 k+1) m(m+1) / 2-k m} .
$$

Thus,

$$
\prod_{n \geq 1}\left(1-q^{n}\right) S_{k}(1)=\sum_{m \in \mathbb{Z}}(-1)^{m} q^{(2 k+1) m(m+1) / 2-k m} .
$$

Applying (48) to the expression in (51), we find

$$
\prod_{n \geq 1}\left(1-q^{n}\right) S_{k}(1)=\zeta_{4 \ell}^{-1} q^{-1 / 8 \ell} \theta\left[\begin{array}{c}
\frac{1}{\ell} \\
1
\end{array}\right](\ell \tau)=\prod_{n \geq 1}\left(1-q^{\ell n}\right)\left(1-q^{\ell n-k}\right)\left(1-q^{\ell n-(k+1)}\right) .
$$

Proof of (46). Again beginning with the definition of $S_{k}(z)$ in (15), we find

$$
\begin{aligned}
\prod_{n \geq 1}\left(1-q^{n}\right) S_{k}(q)= & \sum_{n \geq 0}(-1)^{n} q^{\ell n(n+1) / 2}+\sum_{n \geq 0}(-1)^{n+1} q^{\ell n(n+1) / 2+(\ell-1)(n+1)} \\
& +\sum_{n \geq 0}(-1)^{n+1} q^{\ell n(n+1) / 2+(n+1)}+\sum_{n \geq 0}(-1)^{n} q^{\ell n(n+1) / 2+\ell(n+1)},
\end{aligned}
$$


where (as above) we label the four sums appearing on the right hand side of (52) by $\Sigma_{1}, \Sigma_{2}, \Sigma_{3}$ and $\Sigma_{4}$. Replacing $n$ by $-m-2$ in $\Sigma_{1}$, and also in $\Sigma_{3}$, one finds that

$$
\begin{aligned}
& \Sigma_{1}=\sum_{m \leq-2}(-1)^{m} q^{\ell m(m+1) / 2+\ell(m+1)} \\
& \Sigma_{3}=\sum_{m \leq-2}(-1)^{m+1} q^{\ell m(m+1) / 2+(\ell-1)(m+1)},
\end{aligned}
$$

so that

$$
\begin{aligned}
& \Sigma_{1}+\Sigma_{4}=\sum_{n \in \mathbb{Z}}(-1)^{n} q^{\ell n(n+1) / 2}+1 \\
& \Sigma_{2}+\Sigma_{3}=\sum_{n \in \mathbb{Z}}(-1)^{n+1} q^{\ell n(n+1) / 2+(\ell-1)(n+1)}-1 .
\end{aligned}
$$

We see that the sum $\sum_{n \in \mathbb{Z}}(-1)^{n} q^{\ell n(n+1) / 2}$ appearing in (53) is in fact identically zero: divide the sum into a sum $\sigma_{1}$ over non-negative integers and negative integers $\sigma_{2}$. For the sum $\sigma_{2}$ over negative integers, replace the index of summation $n$ by $-m-1$ to obtain a sum over non-negative integers which becomes $-\sigma_{1}$, so that $\sigma_{1}+\sigma_{2}=\sigma_{1}-\sigma_{1}=0$. Thus,

$$
\begin{aligned}
\sum_{i=1}^{4} \Sigma_{i} & =\sum_{n \in \mathbb{Z}}(-1)^{n+1} q^{\ell n(n+1) / 2+(\ell-1)(n+1)} \\
& =\zeta_{4 \ell}^{-\ell+2} q^{1 / 2-1 / 2 \ell-\ell / 8} \theta\left[\begin{array}{c}
\frac{\ell-2}{\ell} \\
1
\end{array}\right](\ell \tau) \\
& =\prod_{n \geq 1}\left(1-q^{\ell n}\right)\left(1-q^{\ell n-(\ell-1)}\right)\left(1-q^{\ell n-1}\right) .
\end{aligned}
$$

Proof of (47). Using (15) we find

$$
\begin{aligned}
& \prod_{n \geq 1}\left(1-q^{n}\right)\left(S_{k}(1)-q^{k-1} S_{k}\left(q^{2}\right)\right) \\
& =\sum_{n \geq 0}(-1)^{n} q^{(2 k+1) n(n+1) / 2-k n}\left(1-q^{(2 n+1) k}\right) \\
& \quad \quad-\sum_{n \geq 0}(-1)^{n} q^{(2 k+1) n(n+1) / 2+k n+k-1}\left(1-q^{n+1}\right)\left(1-q^{n+2}\right)\left(1-q^{2 n k+3 k}\right) \\
& =\sum_{i=1}^{10} \Sigma_{i},
\end{aligned}
$$

where the ten sums $\Sigma_{i}, 1 \leq i \leq 10$, are formed by expanding the products ( $1-$ $\left.q^{(2 n+1) k}\right)$ and $\left(1-q^{n+1}\right)\left(1-q^{n+2}\right)\left(1-q^{2 n k+3 k}\right)$ appearing in (54). These sums are defined by

$$
\Sigma_{i}:=\varepsilon_{i} \sum_{n \geq 0}(-1)^{n} q^{(2 k+1) n(n+1) / 2+\delta_{i}(n, k)},
$$


where

$$
\delta_{i}(n, k):= \begin{cases}-k n & i=1 \\ k n+k & i=2 \\ k n+k-1 & i=3 \\ k n+k+n & i=4 \\ k n+k+n+1 & i=5 \\ k n+k+2 n+2 & i=6 \\ 3 k n+4 k-1 & i=7 \\ 3 k n+4 k+n & i=8 \\ 3 k n+4 k+n+1 & i=9 \\ 3 k n+4 k+2 n+2 & i=10\end{cases}
$$

and

$$
\varepsilon_{i}:= \begin{cases}1 & i=1,4,5,7,10 \\ -1 & i=2,3,6,8,9 .\end{cases}
$$

From here, we will first pair the sums $\Sigma_{i}$ as we did in the proofs of (45) and (46) above, and then show that $\sum_{i=1}^{10} \Sigma_{i}$ becomes a sum of only three $q$-series which we will then further manipulate.

Lemma 2. With notation as above, we have

$$
\begin{aligned}
& \Sigma_{1}+\Sigma_{5}=1 \\
& \Sigma_{2}+\Sigma_{9}=-q^{k} \\
& \Sigma_{3}+\Sigma_{10}=q^{k}-q^{-1}+\sum_{n \in \mathbb{Z}}(-1)^{n+1} q^{(2 k+1) n(n+1) / 2+k n+k-1} \\
& \Sigma_{4}+\Sigma_{8}=-q^{k-1}+q^{-1}+\sum_{n \in \mathbb{Z}}(-1)^{n} q^{(2 k+1) n(n+1) / 2+k n+k+n} \\
& \Sigma_{6}+\Sigma_{7}=-1+q^{k-1}+\sum_{n \in \mathbb{Z}}(-1)^{n+1} q^{(2 k+1) n(n+1) / 2+k+2 n+k n+2} .
\end{aligned}
$$

Proof of Lemma 2. For the sake of brevity, we indicate in explicit detail how to proceed in order to obtain these identities, leaving the details of the calculation to the reader. First, we consider the sum $\Sigma_{1}+\Sigma_{5}$, and begin by replacing the index of summation $n$ in $\Sigma_{5}$ by $m-1$. After simplifying the power of $q$ appearing, one sees that $\Sigma_{5}=\sum_{m \geq 1}(-1)^{m+1} q^{(2 k+1) m(m+1) / 2-k m}$. Adding to this $\Sigma_{1}$ one visibly sees that all terms cancel except for the term coming from $n=0$ in $\Sigma_{1}$, which is 1 . One proceeds in exactly the same way in considering the sum $\Sigma_{2}+\Sigma_{9}$, first re-indexing $\Sigma_{9}$ by $m-1$. All terms then visibly cancel, except the term coming from $n=0$ in $\Sigma_{2}$, which is $-q^{k}$. For the sum $\Sigma_{3}+\Sigma_{10}$, one replaces the index of summation $n$ in $\Sigma_{10}$ by $-m-3$, and finds after simplifying that $\Sigma_{10}=\sum_{m \leq-3}(-1)^{m+1} q^{(2 k+1) m(m+1) / 2+\delta_{3}(m, k)}$. Thus we see when adding $\Sigma_{3}+\Sigma_{10}$ one obtains the sum $\sum_{m \in \mathbb{Z}}(-1)^{m+1} q^{(2 k+1) m(m+1) / 2+\delta_{3}(m, k)}$ with the exception of the terms coming from $m=-1$ and $m=-2$, which are missing. These two terms are $-q^{k}+q^{-1}$, which we subtract. The sums $\Sigma_{4}+\Sigma_{8}$ and $\Sigma_{6}+\Sigma_{7}$ are handled in an identical manner: one replaces $n$ by $-m-3$ in $\Sigma_{8}$ and $\Sigma_{7}$. Upon doing so one finds the expressions for $\Sigma_{4}+\Sigma_{8}$ and $\Sigma_{6}+\Sigma_{7}$ given in the statement of Lemma 2 , where again the two terms being added to the infinite sum are the negatives of the two terms coming from the indices $m=-1$ and $m=-2$. 
Resuming our proof of (47), by Lemma 2, we have

$$
\sum_{i=1}^{10} \Sigma_{i}=\sum_{n \in \mathbb{Z}}(-1)^{n+1} q^{(2 k+1) n(n+1) / 2}\left(q^{\delta_{3}(n, k)}-q^{\delta_{4}(n, k)}+q^{\delta_{6}(k, n)}\right) .
$$

Expanding $\left(q^{\delta_{3}(n, k)}-q^{\delta_{4}(n, k)}+q^{\delta_{6}(k, n)}\right)$ into the sum on the right hand side of (55), one obtains three infinite sums which we denote by $\sigma_{1}, \sigma_{2}$, and $\sigma_{3}$. It is not difficult to show that $\sigma_{1}+\sigma_{2}=0$ : replace the index of summation $n$ in $\sigma_{1}$ by $n=-r-2$. After simplifying the power of $q$ that appears one sees that $\sigma_{1}=-\sigma_{2}$. Thus, we have

$$
\prod_{n \geq 1}\left(1-q^{n}\right)\left(S_{k}(1)-q^{k-1} S_{k}\left(q^{2}\right)\right)=\sum_{i=1}^{10} \Sigma_{i}=\sigma_{3} .
$$

Using (48), one sees that

$$
\sigma_{3}=q^{-9 / 8 \ell} \zeta_{4 \ell}^{-3} \theta\left[\begin{array}{l}
\frac{3}{\ell} \\
1
\end{array}\right](\ell \tau)=\prod_{n \geq 1}\left(1-q^{\ell n}\right)\left(1-q^{\ell n-(k+2)}\right)\left(1-q^{\ell n-(k-1)}\right) .
$$

Thus we have finished the proof of (47) and hence Proposition 1.

Armed with Proposition 1, we deduce that $r_{\ell, 1}(\tau)$ and $r_{\ell, 2}(\tau)$ are modular units of level $\ell$ by establishing an expression for these functions in terms of Siegel functions. Applying the product expansions obtained in Proposition 1 to the definitions of the definitions of $r_{\ell, 1}(\tau)$ and $r_{\ell, 2}(\tau)$ in (20) and (21), we find

$$
\begin{gathered}
r_{\ell, 1}=(-1)^{k-1} q^{-k(k-1) / 2 \ell} \prod_{n \geq 1} \frac{\left(1-q^{\ell n-k}\right)\left(1-q^{\ell n-(k+1)}\right)}{\left(1-q^{\ell n-1}\right)\left(1-q^{\ell n-(\ell-1)}\right)} \\
r_{\ell, 2}=(-1)^{k-2} q^{-(k+1)(k-2) / 2 \ell} \prod_{n \geq 1} \frac{\left(1-q^{\ell n-(k-1)}\right)\left(1-q^{\ell n-(k+2)}\right)}{\left(1-q^{\ell n-1}\right)\left(1-q^{\ell n-(\ell-1)}\right)} .
\end{gathered}
$$

Next, from (56), (57) and (34) we find the following expressions for the functions $r_{\ell, 1}(\tau), r_{\ell, 2}(\tau):$

$$
\begin{aligned}
& r_{\ell, 1}(\tau)=(-1)^{k-1} e\left(\frac{-k(k-1)}{2 \ell}\right) \prod_{s=0}^{\ell-1} g_{(k / \ell, s / \ell)}(\tau) / g_{(1 / \ell, s / \ell)}(\tau) \\
& r_{\ell, 2}(\tau)=(-1)^{k-2} e\left(\frac{-k(k-2)}{2 \ell}\right) \prod_{s=0}^{\ell-1} g_{(k-1 / \ell, s / \ell)}(\tau) / g_{(1 / \ell, s / \ell)}(\tau) .
\end{aligned}
$$

Thus, by Theorem $\mathrm{K}$, the functions $r_{\ell, 1}$ and $r_{\ell, 2}$ are modular units of level $\ell$. To prove this result for $r_{\ell, j}, 3 \leq j \leq(\ell-3) / 2$, and to prove $i i$, we will make use of Lemma 1 . In what follows for ease of notation, we may omit the variable $\tau$ from the functions $g_{a}(\tau)$ when the context is clear. We compute using the product expansion given for $S_{k}(q)$ given in Proposition 1 as well as the product expansion for the Siegel functions given in (34) that

$$
\Psi_{\ell}(\tau)=-e\left(\frac{k^{2}}{\ell}\right) \prod_{s=0}^{\ell-1} g_{(1 / \ell, s / \ell)}
$$


Next, applying Lemma 1 to the expression for $\Psi_{\ell}$ given in (60) we find

$$
i \Psi_{\ell}(-1 / \tau)=(-1)^{k+1} e\left(k^{2} / \ell\right) \prod_{s=0}^{\ell-1} g_{(s / \ell,-1 / \ell)},
$$

and thus

$$
\gamma_{m}\left(i \Psi_{\ell}(-1 / \tau)\right)=(-1)^{m} e\left(-\frac{k m}{2 \ell}\right) \prod_{s=0}^{\ell-1} g_{(s / \ell,-m / \ell)},
$$

where $\gamma_{m}=\left(\begin{array}{ll}1 & 0 \\ 0 & m\end{array}\right)$. We use the fact that $\sigma_{m}=\left(\begin{array}{cc}0 & 1 \\ -1 & 0\end{array}\right) \gamma_{m}\left(\begin{array}{cc}0 & -1 \\ 1 & 0\end{array}\right)$ and apply Lemma 1 once more to conclude for $r_{\ell, k+1-m}$ as defined in (24),

$$
\begin{aligned}
r_{\ell, k+1-m}(\tau) & =(-1)^{m-1} e\left(-\frac{k(m-1)}{2 \ell}\right) \prod_{s=0}^{\ell-1}\left(\frac{g_{(m / \ell, s / \ell)}}{g_{(1 / \ell, s / \ell)}}\right) \\
& =(-1)^{m-1} \prod_{s=-k}^{k}\left(\frac{g_{(m / \ell, s / \ell)}}{g_{(1 / \ell, s / \ell)}}\right),
\end{aligned}
$$

where the last equality can be derived by using (29). This finishes the proof of $i i$, as well as the proof of $i$ by Theorem $\mathrm{K}$ after verifying the quadratic relations for the Siegel product in (62). We remark that the above calculations verify that the definitions of $r_{\ell, 1}$ and $r_{\ell, 2}$ given in (24), (20) and (21) are consistent.

4.2. Proof of Theorem 1 iii. Theorem 1 iii will follow from Propositions 2, 3 and 4 below.

Proposition 2. For prime $\ell \geq 5$,

$$
\operatorname{Rank} U_{\ell}^{C}=\left|\mathcal{A}_{\ell}\right|-1=\frac{\ell-1}{2}-1 .
$$

Hence,

$$
\operatorname{Rank} U_{\ell}^{C}=\operatorname{Rank} U_{\ell}^{+}
$$

and this rank is the same as that of the cyclotomic unit group $E_{\mathbb{Q}\left(\zeta_{\ell}\right)^{+}}^{C}$.

Proposition 3. For prime $\ell \geq 5$, the groups $U_{\ell}^{C}$ and $U_{\ell}^{+}$are cotorsion free in $U_{\ell}$.

Proposition 4. For prime $\ell \geq 5$,

$$
U_{\ell}^{C} \subseteq U_{\ell}^{+} .
$$

To prove Propositions 2-4, we make the following definition.

Definition. For fixed $\ell=2 k+1 \in \mathbb{N}$, for any $m, 1 \leq m \leq k$, let

$$
g(m)=\prod_{s=0}^{\ell-1} g_{(m / \ell, s / \ell)} .
$$

In the following proposition we will use a fact regarding the order of vanishing at cusps of the functions $g(m)$, where we let $\operatorname{ord}_{\beta} f$ denote the smallest power of $q^{1 / \ell}$ appearing in the Fourier expansion about the cusp $\beta$ of the function $f$. 
Proposition 5. Let $\ell \geq 5$ be prime. Then for cusps $\beta$, $\beta^{\prime}$ not in $\mathcal{A}_{\ell}$, and any integers $m, n, 1 \leq m, n \leq(\ell-1) / 2$,

$$
\operatorname{ord}_{\beta} g(m)=\operatorname{ord}_{\beta^{\prime}} g(n)
$$

Proof of Proposition 5. We make use of the one-to-one correspondence between the cusps of $X(\ell)$ and the Cartan group $C_{2}(\ell) / \pm 1$. The correspondence is given by sending $\gamma \in C_{2}(\ell) / \pm 1$ to the cusp represented by $\gamma \cdot \infty$. (For more detail, see [7], e.g.) Now suppose the cusp $\beta \notin \mathcal{A}_{\ell}$ corresponds to the element $\chi_{\beta}^{-1}:=\left(\begin{array}{ll}a & b \\ c & d\end{array}\right)$ of the Cartan group. Then $\operatorname{ord}_{\beta} g_{a}=\operatorname{ord}_{\infty} g_{a}^{\chi_{\beta}^{-1}}=\operatorname{ord}_{\infty} g_{a \cdot \chi_{\beta}^{-1}}$. Now $\beta \notin A_{\ell}$ implies that $c \not \equiv 0(\bmod \ell)$, for otherwise $\chi_{\beta}^{-1} \in \Gamma_{0}(\ell)$, hence $\pi_{\ell}(\beta)$ is equivalent to $\infty$ on $X_{0}(\ell)$, contradicting our assumption. Using these facts, we compute

$$
\operatorname{ord}_{\beta} g(m)=\sum_{s=0}^{\ell-1} \operatorname{ord}_{\beta} g_{(m / \ell, s / \ell)}=\sum_{s=0}^{\ell-1} \operatorname{ord}_{\infty} g_{(m / \ell, s / \ell)}^{\chi_{\beta}-1}=\sum_{s=0}^{\ell-1} \operatorname{ord}_{\infty} g_{\left(m_{1}(s) / \ell, m_{2}(s) / \ell\right)},
$$

where the parameters $m_{i}(s), 1 \leq i \leq 2$, are given by $(m, s) \chi_{\beta}^{-1}=\left(m_{1}(s), m_{2}(s)\right)=$ $(a m+c s, b m+d s)$. We claim next that the numbers $m_{1}(s)$, as $s$ ranges from 0 to $\ell-1$, must also range over all residues $(\bmod \ell)$. This follows easily from the fact that $c \not \equiv 0(\bmod \ell)$. Finally, we note using (34) that $\operatorname{ord}_{\infty} g_{\left(\frac{a_{1}}{\ell}, \frac{a_{2}}{\ell}\right)}=\frac{\ell}{2} \mathbf{B}_{2}\left(\frac{a_{1}}{\ell}\right)$, and hence is independent of $a_{2}$. Combining these things, we have that $\operatorname{ord}_{\beta} g(m)=$ $\frac{\ell}{2} \sum_{s=0}^{\ell-1} \mathbf{B}_{2}(s / \ell)$, and this number is the same when $m$ is replaced by another integer $n, 1 \leq n \leq(\ell-3) / 2$, and the cusp $\beta \notin A_{\ell}$ is replaced by another cusp $\beta^{\prime} \notin A_{\ell}$.

\section{Proof of Proposition 4.}

Using (62), this follows from Proposition 5.

To prove Proposition 3, we will prove a series of Propositions below.

Proposition 6. For $\ell \geq 5$ prime, the functions $g(m)$ have maximal rank, where $1 \leq m \leq(\ell-1) / 2$. That is, the divisors of the $g(m)$ have rank $\left|\mathcal{A}_{\ell}\right|$.

To prove Proposition 6, we first associate to each $h \in \mathcal{F}_{\ell}$ the order vector $V(h)=$ $\left(\ldots \operatorname{ord}_{\beta} h \ldots\right)$, where $\beta$ runs over all cusps of $X(\ell)$, and the order is taken to be the smallest power of $q^{1 / \ell}$ appearing in the Fourier expansion of $h$ about the cusp $\beta$. If $\rho_{\beta}$ translates the cusp $\beta$ to the standard cusp at infinity, then $\operatorname{ord}_{\beta} h=\operatorname{ord}_{\infty} h^{\rho_{\beta}^{-1}}$, and

$$
V(h)=\left(\ldots \operatorname{ord}_{\infty} h^{\rho_{\beta}} \ldots\right),
$$

where $\rho_{\beta}$ runs over the elements in the Cartan group as in (19). (See [7].) We define the subgroup $C(\ell)^{+}$of the degree two Cartan group $C_{2}(\ell)$ by

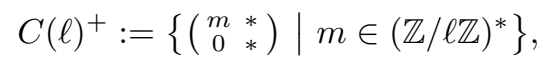

and let $\overline{C(\ell)}{ }^{+}=C(\ell)^{+} /\{ \pm 1\}$. We use the following Proposition to prove Proposition 6. 
Proposition 7. The forms $g(m)$ have maximal rank if and only if for each character $\chi$ of $\overline{C(\ell)}^{+}$we have

$$
\sum_{\beta \in \overline{C(\ell)^{+}}} \chi(\beta) \operatorname{ord}_{\infty} g(\beta) \neq 0
$$

\section{Proof of Propositions 6 and 7.}

We form the $(\ell-1) / 2$ row array with the order vectors $V(g(m))$

$$
\left[\operatorname{ord}_{\alpha} g(m)\right]=\left[\operatorname{ord}_{\infty} g(m)^{\beta}\right]
$$

where for ease of notation we let $\beta$ (rather than $\rho_{\beta}$ ) run over the elements in the full Cartan group $C_{2}(\ell) /\{ \pm 1\}$. Using (67), we form the order $(\ell-1) / 2$ minor with columns determined by $\beta \in \overline{C(\ell)}^{+}$, and denote this minor by

$$
\left[\operatorname{ord}_{\infty} g(m)^{\beta}\right]^{+} .
$$

Proposition 6 will follow from

\section{Proposition 8.}

$$
\operatorname{det}\left[\operatorname{ord}_{\infty} g(m)^{\beta}\right]^{+} \neq 0
$$

\section{Proof of Proposition 8.}

To prove Proposition 8, we will apply the Frobenius determinant relation, which provides a closed form in terms of group characters for the determinant of matrix whose entries are dependent on an Abelian group $G$ and a complex valued function $f$ on $G$.

Proposition 9. (Frobenius determinant relation.) Let $G$ be a finite Abelian group and $\hat{G}$ the group of characters on $G$. For any function $f: G \longrightarrow \mathbb{C}$,

$$
\operatorname{det}_{\alpha, \beta \in G} f\left(\alpha^{-1} \beta\right)=\prod_{\chi \in \hat{G}} \sum_{\alpha \in G} \chi(\alpha) f\left(\alpha^{-1}\right) .
$$

\section{Proof of Proposition 9.}

See [8] Chapter 21, 2 .

Using (66) we apply the Frobenius determinant relation (73) to find

$$
\operatorname{det}\left[\operatorname{ord}_{\infty} g(m)^{\beta}\right]^{+}=\prod_{\chi} \sum_{\beta} \chi(\beta) \operatorname{ord}_{\infty} g(\beta),
$$

as $\chi$ runs over the character group of $\overline{C(\ell)}^{+}$, and $\beta$ over the group. This proves Proposition 7. A short computation using the product expansion for the Siegel functions (34) shows

$$
\operatorname{ord}_{\infty} g(m)=\sum_{s \in \mathbb{Z} / \ell \mathbb{Z}} \operatorname{ord}_{\infty} g_{(m, s)}=\sum_{s \in \mathbb{Z} / \ell \mathbb{Z}} \frac{1}{2} \mathbf{B}_{2}(\langle m / \ell\rangle)=\frac{\ell}{2} \mathbf{B}_{2}(\langle m / \ell\rangle) .
$$


Thus, we have

$$
\sum_{\beta \in \overline{C(\ell)}} \chi(\beta) \operatorname{ord}_{\infty} g(\beta)=\frac{\ell}{2} \sum_{\beta \in \overline{C(\ell)}} \chi(\beta) \mathbf{B}_{2}(\langle\beta / \ell\rangle)=\frac{\ell}{4} \sum_{\beta \in C(\ell)^{+}} \chi(\beta) \mathbf{B}_{2}(\langle\beta \mid \ell\rangle),
$$

which is precisely a multiple of a generalized Bernoulli number of Leopoldt, $\mathbf{B}_{n, \chi}$. The characters on $\overline{C(\ell)}^{+}$extend to even characters on $C(\ell)^{+}$those for which $\chi(-1)=1$, by defining $\chi(\beta)=\chi(-\beta)$. We use the known fact that when $\mathbf{B}_{n}$ and $\chi$ have the same parity, $\mathbf{B}_{n, \chi} \neq 0$. This establishes Proposition 8.

Proof of Proposition 2.

By Theorem 1 ii we have

$$
r_{\ell, k+1-m}=(-1)^{m-1} e\left(-\frac{k(m-1)}{2 \ell}\right) g(m) / g(1)
$$

where $2 \leq m \leq k$. For any fixed $n$, the quotient $g(m) / g(n)$ is a modular unit (up to appropriate constant) for all $m \neq n$. The modular units $r_{\ell, k+1-m}$ have maximal possible rank $\left|\mathcal{A}_{\ell}\right|-1$ because the functions $g(m)$ have maximal possible rank $\left|\mathcal{A}_{\ell}\right|$ by Proposition 6 . This gives Proposition 2.

\section{Proof of Proposition 3.}

The result follows easily for the group $U_{\ell}^{+}$. For suppose for some $h \in U_{\ell}$, $h^{k} \in U_{\ell}^{+}$for some $k \in \mathbb{Z}^{+}$. Then $\operatorname{ord}_{\beta} h^{k}=k \operatorname{ord}_{\beta} h$, so that $\operatorname{ord}_{\beta} h=0$ for all $\beta \notin \mathcal{A}_{\ell}$. Thus $h \in U_{\ell}^{+}$. For the group $U_{\ell}^{C}$, we again suppose for some $h \in U_{\ell}$, $h^{k} \in U_{\ell}^{C}$. It is enough to establish the result for prime $k$, by factoring. We may write

$$
h^{k}=\prod_{m} g(m)^{n(m)} .
$$

To proceed, we will use a result found in [7]. Although this result is not stated as a Lemma in [7], we do so here.

Lemma 3. ([7] p.91) Let $k$ be a prime number. If

$$
h^{k}=\prod g_{a}^{n(a)}
$$

and $\ell a=\ell b=0$, then $k$ divides $n(a)-n(b)$.

For each $g_{(m / \ell, s / \ell)}$ in the product for $h^{k}$, we pair the element $g_{(0, s / \ell)}$, and write their respective exponents $n(m, s)$ and $n(0, s)$. Here $n(0, s)=0$ as $g_{(0, s / \ell)}$ does not appear in the product, and we will write $n(m, s)=n(m)$. Lemma 3 shows that for each $g_{(m / \ell, s / \ell)}$ appearing in the product for $h^{k}$, the exponent $k$ divides $n(m, s)=n(m)$, so that $h$ has an expression

$$
h=\prod_{m} g(m)^{n^{\prime}(m)} .
$$

The fact that $\sum_{m} n(m)=0$ shows that $\sum_{m} n^{\prime}(m)=0$, and since $h \in U_{\ell}$, we must have $h \in U_{\ell}^{C}$. Thus, we have the desired characterization of the modular units $U_{\ell}^{C}$. 


\section{Acknowledgements}

The author wishes to express her gratitude to Bill Duke and also the referee for many detailed and helpful comments on earlier versions of this paper.

\section{References}

[1] G. Andrews, An analytic proof of the Rogers-Ramanujan-Gordon identities, Amer. J. Math. 88 (1966), 844-846.

[2] G. Andrews, An introduction to Ramanujan's 'lost' notebook, Amer. Math. Monthly 86 (1979), no. 2, 89-108.

[3] W. Duke, Continued fractions and modular functions, Bull. Amer. Math. Soc. (N.S.) 42 (2005), 137-162.

[4] H. Farkas and I. Kra, Theta Constants, Riemann Surfaces and the Modular Group, AMS Graduate Studies in Mathematics, 37. American Mathematical Society, Providence, (2001).

[5] A. Folsom, Class invariants and cyclotomic unit groups from special values of modular units, Journal des Théorie des Nombres Bordeaux, (20) no. 2 (2008), 289-325.

[6] J. Jorgenson and S.G. Krantz, The mathematical contributions of Serge Lang, Notices Amer. Math. Soc. 54 (2007), 476-497.

[7] D. S Kubert and S. Lang, Modular Units, Grundlehren der Mathematischen Wissenschaften [Fundamental Principles of Mathematical Science], 244. Springer-Verlag, New York-Berlin, (1981).

[8] S. Lang, Elliptic Functions. Addison-Wesley Publishing Co., Reading, MA, (1973).

[9] L.J. Rogers, Second Memoir on the Expansion of Certain Infinite Products, Proc. London Math. Soc. 25 (1894), 318-343.

[10] L.J. Rogers and S. Ramanujan, Proof of certain identities in combinatory analysis, Cambr. Phil. Soc. Proc. 19 (1919), 211-216.

[11] I. Schur, Ein Beitrag zur additiven Zahlentheorie und zur Theorie der Kettenbrüche, Sitzungsber. Preuss. Akad. Wiss. Phys.-Math. Klasse (1917) 302-321.

[12] A. Selberg, Über einige arithmetische Identitäten, Avh. Norske Vidensk. Akad. Oslo, I 1936, Nr. 8, 23 S.(1936); reprinted in Collected Papers, Vol. I, Springer-Verlag, Berlin, (1989).

Department of Mathematics, UClA, Box 951555 Los Angeles, CA 90095

E-mail address: alfolsom@math.ucla.edu

Current address: University of Wisconsin Mathematics, 480 Lincoln Dr. Madison, WI 53706

E-mail address: folsom@math.wisc.edu 